\title{
Geometric phase effects in dynamics near conical intersections: Symmetry breaking and spatial localization
}

\author{
Ilya G. Ryabinkin and Artur F. Izmaylov \\ Department of Physical and Environmental Sciences, \\ University of Toronto Scarborough, Toronto, Ontario, M1C 1A4, \\ Canada; and Chemical Physics Theory Group, Department of Chemistry, \\ University of Toronto, Toronto, Ontario, M5S 3H6, Canada
}

(Dated: February 22, 2018)

\begin{abstract}
We show that finite systems with conical intersections can exhibit spontaneous symmetry breaking which manifests itself in spatial localization of eigenstates. This localization has a geometric phase origin and is robust against variation of model parameters. The transition between localized and delocalized eigenstate regimes resembles a continuous phase transition. The localization slows down the low-energy quantum nuclear dynamics at low temperatures.
\end{abstract}

A conical intersection (CI) of several electronic states is one of the most common structural motifs in molecules where the Born-Oppenheimer (BO) approximation breaks down [1, 2]. Due to energetic proximity of potential energy surfaces, nuclear motion near CI triggers electronic transitions. These transitions are not the only effect that CIs produce: parametric evolution of the eigenstates of the electronic Hamiltonian along a closed path encircling a locus of CI gives rise to an extra (-1) phase factor accumulated by the intersecting electronic eigenstates [3, 4]. This additional phase, termed the geometric phase (GP) [4 6] does not depend on a size or a shape of the encircling loop, provided that no other degeneracies are enclosed. GP also affects the nuclear motion, because changing the sign of the electronic wavefunction will necessarily change the sign of the nuclear wavefunction in order for the total wavefunction to be single-valued. GP effects were found to be crucial for modeling vibrational spectra of JahnTeller distorted compounds (e.g., $\mathrm{Na}_{3}$ ) [7 9] and cross sections in low-energy atom-molecule reactive scattering (e.g., $\mathrm{H}+\mathrm{O}_{2}$ ) 10 14.

In this Letter, we report yet another remarkable GP effect: spontaneous symmetry breaking that manifests itself in spatial localization of low-energy eigenstates. It is common in quantum mechanics that low-lying eigenstates are delocalized over all energetically-accessible regions. Delocalization lowers the kinetic energy of a system and, if it is not counteracted by the potential, lowers the total energy. However, in the presence of GP some eigenstates are found to be immune to delocalization even though the potential does not counteract. Although this localization can be seen as a consequence of destructive interference between different tunneling paths connecting energetically-accessible regions in systems with CI 1519], we show that the destructive interference alone is not sufficient for the localization.

We consider a generic two-state ("full") model exhibiting CI along with its two single-surface approximations: $\mathrm{BO}$ and $\mathrm{BO}+\mathrm{GP}$. The $\mathrm{BO}$ model neglects GP completely, whereas the $\mathrm{BO}+\mathrm{GP}$ model uses a position-dependent
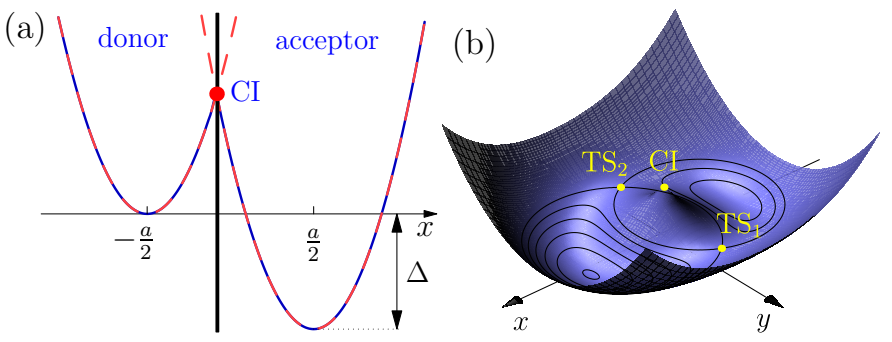

Figure 1. a) Diabatic potentials (red dashed lines) $V_{11}$ and $V_{22}$, and the lowest adiabatic potential $W_{-}$(blue solid line), in $y=0$ section. The thick black line separates donor and acceptor wells. b) The lowest adiabatic potential $W_{-}$with two symmetric transition states labelled as $\mathrm{TS}_{1}$ and $\mathrm{TS}_{2}$.

phase factor $e^{i \theta(\mathbf{x})}$ that changes the sign of a nuclear wavefunction upon encircling CI [5, 20]. The two-state model includes both GP and non-adiabatic transitions, therefore comparing results from all three models allows us to isolate and quantify pure GP effects.

As we show below, the spinor symmetry of the full model is preserved by the $\mathrm{BO}+\mathrm{GP}$ model but is lost in the $\mathrm{BO}$ approximation. The spinor symmetry gives rise to degeneracy of the ground state for some values of model parameters and thus produces the quantum phase transition [21]. Small variations of parameters can lift the degeneracy, but associated localization does not disappear. Further parameter variation eventually destroys the localization, and critical values for the parameters form a phase diagram. The phase diagram qualitatively explains observed differences in nuclear dynamics at low energies with and without GP.

Model. - In a diabatic representation, the Hamiltonian of the two-state CI model is

$$
\hat{H}=\hat{T}_{N} \mathbf{1}_{2}+\left(\begin{array}{ll}
V_{11} & V_{12} \\
V_{12} & V_{22}
\end{array}\right),
$$

where $\hat{T}_{N}$ is the nuclear kinetic energy operator, $V_{11}$ and $V_{22}$ are diabatic potentials represented by identical $2 \mathrm{D}$ parabolas shifted in space and in energy (Fig. 1, and 
coupled by the $V_{12}$ potential $(\hbar=1)$

$$
\begin{aligned}
& V_{11}=\frac{\omega_{1}^{2}}{2}\left(x+\frac{a}{2}\right)^{2}+\frac{\omega_{2}^{2}}{2} y^{2}+\Delta / 2, \quad V_{12}=c y, \\
& V_{22}=\frac{\omega_{1}^{2}}{2}\left(x-\frac{a}{2}\right)^{2}+\frac{\omega_{2}^{2}}{2} y^{2}-\Delta / 2 .
\end{aligned}
$$

In spite of its simplicity, $N$-dimensional generalization of this 2D model was successfully applied to modeling vibronic spectra of real molecules with CIs [22, 23]. Diagonalization of the two-state potential matrix in Eq. (1) gives the following adiabatic potentials

$$
W_{ \pm}=\frac{1}{2}\left(V_{11}+V_{22}\right) \pm \frac{1}{2} \sqrt{\left(V_{11}-V_{22}\right)^{2}+4 V_{12}^{2}} .
$$

We focus on a lower-surface Hamiltonian $\hat{H}_{-}=\hat{P}^{2} / 2+$ $W_{-}$(Figs. 1 $\left.1 \mathrm{a}-\mathrm{b}\right)$, which governs the dynamics in the $\mathrm{BO}$ and $\mathrm{BO}+\mathrm{GP}$ approximations. The $\mathrm{BO}$ and $\mathrm{BO}+\mathrm{GP}$ models differ in form of $\hat{P}: \hat{P}^{\mathrm{BO}}=-i \nabla$ for the $\mathrm{BO}$ model $\left[\hat{H}_{-}^{\mathrm{BO}}=\hat{H}_{-}\left(\hat{P}^{\mathrm{BO}}\right)\right]$, and $\hat{P}^{\mathrm{GP}}=-i e^{-i \theta} \nabla e^{i \theta} \equiv-i \nabla+\nabla \theta$ for the $\mathrm{BO}+\mathrm{GP}$ model $\left[\hat{H}_{-}^{\mathrm{GP}}=\hat{H}_{-}\left(\hat{P}^{\mathrm{GP}}\right)\right]$. The latter $\hat{P}^{\mathrm{GP}}$ identity shows that one can still work with singlevalued wavefunctions even in the presence of GP at expense of an extra term in definition of the canonical momentum [24]. $\theta=\theta(x, y)$ is defined as the mixing angle of the two-state unitary transformation that diagonalizes the potential matrix in Eq. (11) 25]

$$
\theta(x, y)=\frac{1}{2} \arctan \frac{2 V_{12}}{V_{11}-V_{22}}=\frac{1}{2} \arctan \frac{\gamma y}{x-b},
$$

where $b=\Delta /\left(\omega_{1}^{2} a\right)$ is the $x$-coordinate of the CI point, and $\gamma=2 c / \omega_{1}^{2} a$ is dimensionless coupling strength.

Symmetry and spectrum degeneracies. - For $\Delta=0$ and arbitrary values of other parameters, the potential $W_{-}$possesses $C_{2 v}$ symmetry, which is also the symmetry of the BO Hamiltonian $\hat{H}_{-}^{\mathrm{BO}}$. In contrast, $\hat{H}_{-}^{\mathrm{GP}}$ and $\hat{H}$ have the double group symmetry $C_{2 v}^{\dagger}[26$ ] that adds to the $C_{2 v}$ elements an extra rotation by $2 \pi(R)$. $R$ acts nontrivially only on the double-valued eigenfunctions of $\hat{H}_{-}^{\mathrm{GP}}$ and two-component eigenfunctions of $\hat{H} . \quad C_{2 v}^{\dagger}$ is a non-Abelian group, and all eigenfunctions of $\hat{H}_{-}^{\mathrm{GP}}$ and $\hat{H}$ transform according to its two-dimensional irreducible spinor representation $E_{1 / 2}$, giving rise to a doublydegenerate spectrum. By allowing $\Delta \neq 0$, we lower the symmetry to $C_{s}$ for $\hat{H}_{-}^{\mathrm{BO}}$ and to $C_{s}^{\dagger}$ for $\hat{H}_{-}^{\mathrm{GP}}$ and $\hat{H}$. Both $C_{s}$ and $C_{s}^{\dagger}$ are Abelian, and thus, there are no systematic degeneracies in spectra of all three Hamiltonians any more. Correspondingly, the doubly-degenerate eigenvalues of $\hat{H}_{-}^{\mathrm{GP}}$ and $\hat{H}$ split as $E_{1 / 2}=B_{1} \oplus B_{2}$ [27].

Eigenstate localization and symmetry breaking.Ground-state degeneracy in the BO+GP and full models leads to the spontaneous localization of the lowest eigenstates that has no analogs in the $\mathrm{BO}$ model. It is well-known that the eigenstates of $\hat{H}_{-}^{\mathrm{BO}}$ with the symmetric arrangement of wells $(\Delta=0)$ will be delocalized over the wells [28]. For high barriers, the low-lying eigenstates of $\hat{H}_{-}^{\mathrm{BO}}$ have a group structure where states within a group are separated by a small energy gap, while different groups are separated by large energy gaps. The ground and first excited states $\Psi_{1,2}^{\mathrm{BO}}$ of $H_{-}^{\mathrm{BO}}$ are delocalized functions corresponding to the lowest energy group. By rotating within the $\Psi_{1,2}^{\mathrm{BO}}$ subspace, one can obtain function $\Phi=\Psi_{1}^{\mathrm{BO}} \sin \eta+\Psi_{2}^{\mathrm{BO}} \cos \eta$ that is localized in the donor well. However, $\Phi$ is not an eigenfunction of $H_{-}^{\mathrm{BO}}$, and thus, it will escape from the donor well within a time period inversely proportional to the energy gap between the $\Psi_{1,2}^{\mathrm{BO}}$ eigenenergies.

To consider the full diabatic problem [Eq. (1)] we introduce the lowest eigenstates $\Phi_{\mathrm{D}}$ and $\Phi_{\mathrm{A}}$ of the donor and acceptor Hamiltonians $\hat{H}_{\mathrm{D}}=\hat{T}_{N}+V_{11}$ and $\hat{H}_{\mathrm{A}}=$ $\hat{T}_{N}+V_{22}$, respectively. Within the full problem, vectors $\left(\Phi_{\mathrm{D}}, 0\right)^{\dagger}$ and $\left(0, \Phi_{\mathrm{A}}\right)^{\dagger}$ cannot interact via $V_{12} \sigma_{x}$ because both $\Phi_{\mathrm{D}}$ and $\Phi_{A}$ are even with respect to $y \rightarrow-y$, while $V_{12}$ is odd. Therefore, true lowest eigenfunctions of the full problem $\Psi_{1}^{\text {full }}$ and $\Psi_{2}^{\text {full }}$ are dominated by the vectors $\left(\Phi_{\mathrm{D}}, 0\right)^{\dagger}$ and $\left(0, \Phi_{\mathrm{A}}\right)^{\dagger}$, while admixture of higher eigenfunctions of $\hat{H}_{\mathrm{D}}$ and $\hat{H}_{\mathrm{A}}$ is suppressed by an energy gap of at least $\omega_{2}$. Owing to the degeneracy of the full problem spectrum, one can always rotate $\Psi_{1,2}^{\text {full }}$ into a pair of localized eigenfunctions $\Psi_{\mathrm{D}, \mathrm{A}}^{\text {full }}$ that are close to the $\left(\Phi_{\mathrm{D}}, 0\right)^{\dagger}$ and $\left(0, \Phi_{\mathrm{A}}\right)^{\dagger}$ states. Similarly, rotating the lowest doublet components $\Psi_{1,2}^{\mathrm{GP}}$ of $\hat{H}_{-}^{\mathrm{GP}}$ also produces spatially localized eigenstates $\Psi_{D, A}^{\mathrm{GP}}$. Thus, the spectral degeneracy leads to localization of the lowest eigenstates and spontaneous symmetry breaking in both full and $\mathrm{BO}+\mathrm{GP}$ models.

In the $\Delta \neq 0$ case, although the degeneracy of the spectra in the full and $\mathrm{BO}+\mathrm{GP}$ models is lifted, the localization of low-lying states survives in both models provided that $\Delta$ is not "too large" $\left(\Delta<\omega_{i}\right)$. For the full model, $\Delta \neq 0$ is equivalent to introducing $\sigma_{z} \Delta / 2$ perturbation to the $\Delta=0$ Hamiltonian. This diagonal perturbation does not couple the localized eigenstates $\Psi_{\mathrm{D}, \mathrm{A}}^{\text {full }}$ to the first order, but only lifts the degeneracy by changing energies of these states. Higher-order contributions from states other than $\Psi_{\mathrm{D}, \mathrm{A}}^{\mathrm{full}}$ are energetically suppressed. In the GP+BO model, we start with a localized state of the donor well for the $\Delta=0$ problem $\Psi_{\mathrm{D}}^{\mathrm{GP}}$ and consider its dynamics in the $\Delta \neq 0$ case. Two minimal energy paths to the acceptor minimum are available for $\Psi_{\mathrm{D}}^{\mathrm{GP}}$ via transition states $\mathrm{TS}_{1}$ and $\mathrm{TS}_{2}$ (Fig. 1b). Phases acquired by the wave packet along these paths are close to $e^{i \pi / 2}=i$ and $e^{-i \pi / 2}=-i$. Therefore, the interference between the parts of the wave packet that take different paths is destructive and leads to forming a nodal $y=0$ line in the wave packet [15, 29]. A wave function with an extra node has higher energy (approximately by $\omega_{2}$ ), and thus, the $\Psi_{\mathrm{D}}^{\mathrm{GP}}$ escape from the donor well is energetically suppressed when $\Delta<\omega_{2}$.

These qualitative considerations break down for suffi- 

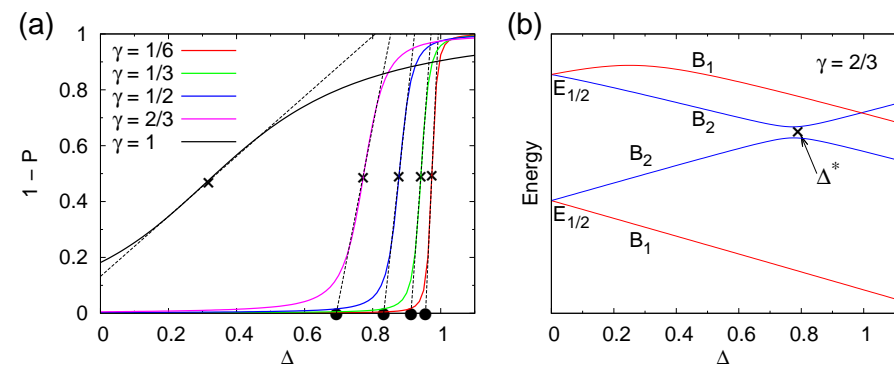

Figure 2. a) Delocalization degree $(1-P)$ for the $\hat{H}_{-}^{\mathrm{GP}}$ first excited state as a function of $\Delta$. Crosses mark inflection points, dashed lines are tangent lines at the inflection points. Filled circles separate the regions of slow and fast growth of $P . \mathrm{b}$ ) Correlation diagram for low-energy eigenstates of $\hat{H}_{-}^{\text {GP }}$. The cross marks the energy gap minimum of two states in the avoided-crossing and corresponds to the inflection point of the $1-P(\gamma=2 / 3)$ curve on panel (a).

ciently large $\Delta$, therefore we perform numerical simulations within the $\mathrm{BO}+\mathrm{GP}$ approximation to find critical values of $\Delta$ when localization disappears. For simplicity of the subsequent discussion only the isotropic case $\omega_{1}=\omega_{2}=1$ will be considered, while its generalization for the $\omega_{1} \neq \omega_{2}$ case is straightforward. Also, instead of an absolute value of the coupling $c$ we use the dimensionless parameter $\gamma$.

We separate donor and acceptor wells (Fig. Th) using a projector operator $\hat{P}(x, y)$ that equals $1(0)$ if $(x, y)$ is in the donor(acceptor) well. For an eigenstate $\Psi$ the average value $P=\langle\Psi|\hat{P}| \Psi\rangle$ provides a quantitative measure of the $\Psi$ localization. In a doubly degenerate case $\Psi=$ $\Psi_{1} \cos \eta+\Psi_{2} \sin \eta$, where $\Psi_{1,2}$ are orthogonal components of the eigen-subspace, we define the $\Psi$ localization as $\max _{\eta} P$.

Figure 2 a presents the delocalization degree $1-P$ of the first excited state as a function of $\Delta[30]$. The delocalization quickly raises to 1 when $\Delta$ (for a given $\gamma$ ) passes through a certain critical value $\Delta^{*}(\gamma)$. This behavior can be explained by considering the eigenvalue correlation diagram (Fig. 2b): at $\Delta^{*}$ the first excited state exhibits an avoided-crossing with the second excited state of the same symmetry but localized in the acceptor well. The donor state restores its localization beyond the critical point when it becomes the second excited state. However, after that point its energy grows beyond the region relevant to low-energy dynamics.

Based on the shape of $1-P$ curves (Fig. 2 2 ), we propose two definitions of $\Delta^{*}: 1$ ) the inflection point of the $1-P$ curve, and 2) the intersection of the tangent line at the inflection point with the $\Delta$ axis. Since we consider finite systems where true continuous phase transitions between localized and delocalized states are impossible, these two definitions of $\Delta^{*}$ give different estimates for transition points. We put both critical values of $\Delta^{*}$ for different $\gamma$ on the same plot and obtain the phase dia-

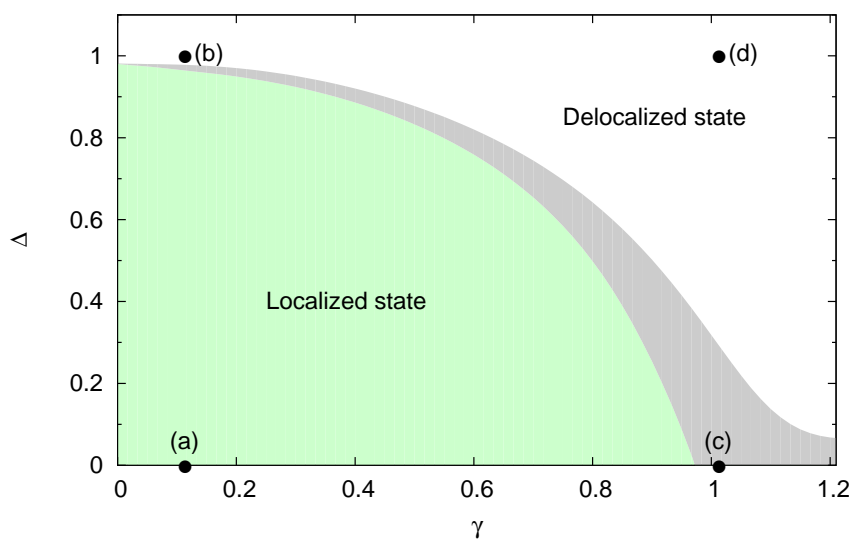

Figure 3. Phase diagram based on the critical values of $\Delta$ from Fig. 2a: the inflection points of the $1-P$ curves (greywhite boundary), the intersections of the tangent lines with the $\Delta$ axis (green-grey boundary). Black dots (a-d) mark the parameter values for which nuclear dynamics is presented in Fig. 4(a-d).

gram for $\hat{H}_{-}^{\mathrm{GP}}$ in Fig. 3. Colored areas correspond to a range of couplings $(\gamma)$ and acceptor shifts $(\Delta)$ for which the donor - despite unfavorable energetics - still supports the localized eigenstate.

Nuclear dynamics. - To elucidate the impact of GP and localization on the dynamics, we simulate the probability transfer $P(t)=\operatorname{tr}\{\hat{\rho}(t) \hat{P}\}$ starting from the Boltzmann density of the donor Hamiltonian $\hat{H}_{\mathrm{D}}$ at temperature $T$ (simulation details are given in Ref. 31). The localization of eigenstates in the presence of GP has a large impact on the nuclear dynamics. At $T=0$, in the presence of the localization in $\hat{H}_{-}^{\mathrm{GP}}$, our initial state can be close to stationary by almost coinciding with a single eigenstate of $\hat{H}_{-}^{\mathrm{GP}}$. In contrast, in the BO model where the localization is impossible, the initial wave packet is predominantly a superposition of two lowest $\mathrm{BO}$ eigenstates. Indeed, at $\gamma=0.1$ and $\Delta=0$ [point (a) in Fig. [3] both full and $\mathrm{BO}+\mathrm{GP}$ models demonstrate complete suppression of the tunnelling, while the $\mathrm{BO}$ model produces unit size coherent oscillations according to the Rabi twolevel model (Fig. 4h). When we are slightly above the edge of stability of the localized phase in the $\mathrm{BO}+\mathrm{GP}$

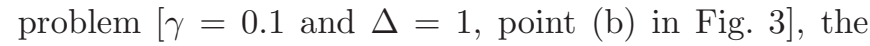
dynamics of all three models are significantly different, and the $\mathrm{BO}+\mathrm{GP}$ dynamics is faster than those of the full model (Fig. 40). The analysis of the eigenvalue correlation diagram for the full model reveals that the corresponding critical $\Delta^{*}$ for $\gamma=0.1$ is slightly larger than that of the $\mathrm{BO}+\mathrm{GP}$ model. Thus, the dynamics represents only the onset of the localized state decay in the full model, while for the BO+GP model the localized state is already unstable. Except for a rather narrow range of $\Delta$ 's the full and $\mathrm{BO}+\mathrm{GP}$ models are quite similar (Fig. 目,c,d). The role of non-adiabatic transitions is 

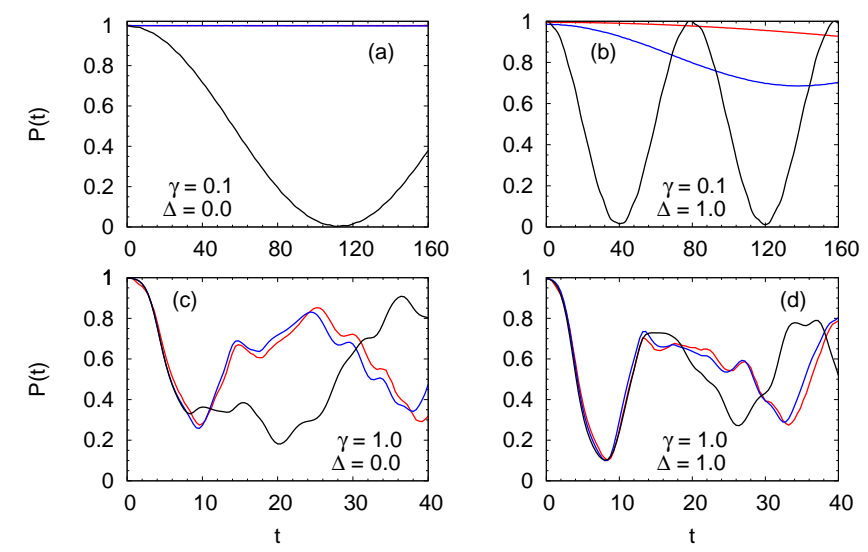

Figure 4. Population transfer dynamics for $T=0$ : the full model (red), the BO+GP model (blue), the BO model (black).
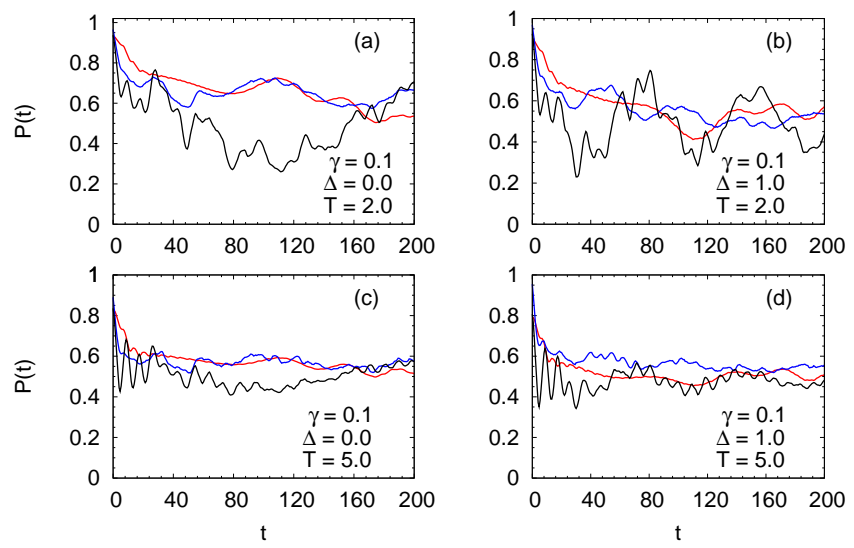

Figure 5. Population transfer dynamics for finite T's: the full model (red), the BO+GP model (blue), the BO model (black).

indeed small, and all observed effects can be attributed to the presence of GP. Finally, in the delocalized region [point (d) in Fig. 3] where the initial localized state is unrepresentable as a single eigenstate, all three models demonstrate almost quantitatively similar dynamics with quick and profound population transfer (Fig. 4 $\mathrm{d}$ ).

Our non-zero $T$ set-up corresponds to quick thermalization of the initial state by environment followed by system dynamics that does not account for interaction with environment. Temperature averaging includes several states that may have different degrees of localization. If we populate levels with a similar localization pattern, $T \neq 0$ dynamics preserves the qualitative features of that for $T=0$ ( $c f$. Fig. 4 a-b with Fig. 5 a-b), whereas differences in the localization character of populated levels create differences in dynamics ( $c f$. Fig. 4a with Fig. 5a). Increasing temperature even more will eventually populate enough levels to wash out all state-specific dynamical features (Fig. 5i-d).
Extensions of the model. - Understanding dynamics with GP for more complex Hamiltonians can be accomplished via perturbative consideration of the corresponding two-state problem. Two main factors are: the selection rules for the coupling matrix elements between diabatic vibrational levels, and energy differences between coupled levels. For more general coupling potentials $V_{12} \sim y^{n} \sigma_{x}$ odd $n$ 's are expected to produce similar effects to those of $n=1$ as they can support the spinor symmetry, while even $n$ 's cannot and their behavior is closer to the case of a constant coupling potential. Other ways to modify selection rules also include introducing different frequencies for the donor and acceptor, non-orthogonal tuning and coupling coordinates, Dushinsky rotation or anharmonicity of wells. All these modifications unless they are too strong can be analyzed perturbatively.

In conclusion, we investigated dynamical consequences of the spontaneous symmetry breaking in the twodimensional CI model. The naive BO approximation cannot capture the correct spinor symmetry of the problem and breaks down qualitatively even for the nuclear dynamics in regions that are far from the CI. Introducing GP explicitly into the nuclear wavefunction restores the symmetry and the associated symmetry breaking. The latter leads to the spatial localization of vibronic eigenstates and freezes the inter-well dynamics. Owing to the topological character of this effect one can see this as an example of a topological insulating state 32] in a finite system. Variation of parameters can lower the overall symmetry and remove the symmetry breaking; however, the localization of the eigenstates and its dynamical consequences persist in some range.

We thank L. A. Pachon, T. V. Tscherbul, S. Wittington, V. V. Albert, J. Endicott, and P. Brumer for helpful discussions and comments on the manuscript. This work was supported by NSERC of Canada through the Discovery Grants Program.

[1] A. Migani and M. Olivucci, in Conical Intersection Electronic Structure, Dynamics and Spectroscopy, edited by W. Domcke, D. R. Yarkony, and H. Köppel (World Scientific, New Jersey, 2004) p. 271.

[2] D. R. Yarkony, Rev. Mod. Phys. 68, 985 (1996)

[3] H. C. Longuet-Higgins, U. Opik, M. H. L. Pryce, and R. A. Sack, P. Roy. Soc. A - Math. Phy. 244, 1 (1958).

[4] M. V. Berry, P. Roy. Soc. A - Math. Phys. 392, 45 (1984)

[5] C. A. Mead and D. G. Truhlar, J. Chem. Phys. 70, 2284 (1979).

[6] M. V. Berry, P. Roy. Soc. A - Math. Phys. 414, 31 (1987)

[7] B. Kendrick, Phys. Rev. Lett. 79, 2431 (1997).

[8] F. S. Ham, Phys. Rev. Lett. 58, 725 (1987)

[9] M. S. Child, in The Role of Degenerate States in Chemistry Advances in Chemical Physics, Vol. 124, edited by M. Baer and G. D. Billing (John Wiley \& Sons, Inc., 
2003) pp. 1-38.

[10] B. Kendrick, J. Chem. Phys. 104, 7475 (1996)

[11] B. Kendrick, J. Chem. Phys. 104, 7502 (1996)

[12] B. K. Kendrick, J. Chem. Phys. 112, 5679 (2000).

[13] B. K. Kendrick, J. Phys. Chem. A 107, 6739 (2003)

[14] J. C. Juanes-Marcos, S. C. Althorpe, and E. Wrede, Science 309, 1227 (2005)

[15] J. Schön and H. Köppel, J. Chem. Phys. 103, 9292 (1995).

[16] A. Garg, Europhys. Lett. 22, 205 (1993)

[17] A. Garg, Europhys. Lett. 50, 382 (2000)

[18] E. Keçecioğlu and A. Garg, Phys. Rev. B 63, 064422 (2001)

[19] D. Stelitano, Phys. Rev. D 51, 5876 (1995).

[20] C. A. Mead, Rev. Mod. Phys. 64, 51 (1992)

[21] S. Sachdev, Quantum phase transitions, 2nd ed. (Cambridge University Press, 2011).

[22] H. Köppel, W. Domcke, and L. S. Cederbaum, Adv. Chem. Phys. 57, 59 (1984).

[23] G. A. Worth and L. S. Cederbaum, Ann. Rev. Phys. Chem. 55, 127 (2004).

[24] C. Wittig, Phys. Chem. Chem. Phys. 14, 6409 (2012).

[25] Care must be taken when $\operatorname{arguments}$ of $\arctan (y / x)$ cross the coordinate axes. A convenient choice is to allow the functional discontinuities from $\pi$ to $-\pi$ upon crossing the negative $x$ axis in the counterclockwise direction.

[26] S. L. Altmann, Rotations, Quaternions, and Double Groups, Dover Books on Mathematics Series (Dover Publications, Incorporated, 2005).

[27] We use $B_{1}$ and $B_{2}$ representations of the $C_{2 v}$ group to label the corresponding representations of the isomorphic double-group $C_{s}^{\dagger}$.

[28] S. Takada and H. Nakamura, J. Chem. Phys. 100, 98 (1994).

[29] J. Schön and H. Köppel, Chem. Phys. Lett. 231, 55 (1994)

[30] The ground state is trivially localized for $\Delta \neq 0$ since we always assume that the acceptor is lower in energy than the donor.

[31] See supplemental material at http://link.aps.org/supplemental/XXX for details on numerical simulations.

[32] M. Z. Hasan and C. L. Kane, Rev. Mod. Phys. 82, 3045 (2010). 